\title{
Heterologous expression of a novel Poa pratensis gibberellin 2-oxidase gene, $P p G A 20 x$, caused dwarfism, late flowering, and increased chlorophyll accumulation in Arabidopsis
}

\author{
P.-H TAN ${ }^{1,2}$, L. ZHANG ${ }^{1}$, S.-X. YIN ${ }^{1 *}$, and K. TENG ${ }^{2 *}$ \\ Turfgrass Research Institute, Beijing Forestry University, Beijing 100083, P.R. China ${ }^{1}$ \\ Beijing Research and Development Center for Grass and Environment, \\ Beijing Academy of Agriculture and Forestry Sciences, Beijing 100097, P.R. China ${ }^{2}$
}

\begin{abstract}
Gibberellin 2-oxidases (GA2oxs) irreversibly convert bioactive gibberellins (GAs) and their immediate precursors into inactive GAs via $2-\beta$ hydroxylation and so regulate gibberellin content in plants. However, to the best of our knowledge, little has been known about the GA2oxs and its function in cool season turfgrass Poa pratensis. In this study, rapid amplification of cDNA end (RACE) was employed to isolate PpGA2ox from P. pratensis. The open reading frame of $P p G A 2 o x$ was $1047 \mathrm{bp}$ in length, corresponding to 348 amino acids. PpGA2ox was localized in both nucleus and cytoplasm. The expression of $P p G A 2 o x$ could be up-regulated by $10 \mu \mathrm{M}$ gibberellic acid, $5 \mu \mathrm{M}$ methyl jasmonate, or $10 \mu \mathrm{M}$ indole-3-acetic acid. In addition, its native promoter could drive GUS expression in both leaf apex and shoot apical region. Moreover, overexpression of PpGA2ox in Arabidopsis led to GA-deficiency leading to dwarf phenotype, delayed flowering time, and increased chlorophyll content. Our study suggests that PpGA2ox could be a candidate gene for breeding new cultivars of $P$. pratensis.
\end{abstract}

Additional key words: gibberellic acid, indole-3-acetic acid, methyl jasmonate, transgenic plants.

\section{Introduction}

Bioactive gibberellins (GAs) play crucial roles in plant growth and development including stem elongation, leaf expansion, and flower development (Yamaguchi 2008, Hedden and Thomas 2012, Yin et al. 2017). In plants, geranylgeranyl diphosphate, a common $\mathrm{C}_{20}$ precursor for diterpenoids, is converted into GAs by several reactions using terpene synthases, cytochrome $\mathrm{P}_{450}$ monooxygenases, and 2-oxo-glutarate-dependent dioxygenases (Yamaguchi 2008). To achieve a dynamic balance, plants have also evolved an effective deactivation system and GA2-oxidases (GA2oxs) irreversibly inactivate GAs and its immediate precursors via $2-\beta$ hydroxylation (Hedden and Phillips 2000, Wuddineh et al. 2015).

Recently, GA2oxs have been increasingly characterrized in various plant species including Prunus salicina (El-Sharkawy et al. 2012), Panicum virgatum (Wuddineh et al. 2015), Camellia lipoensis (Xiao et al. 2016), and Brassica napus (Yan et al. 2017). Heterologous overexpression of plum GA2ox in Arabidopsis thaliana causes characteristic GA deficient phenotypes including smaller leaves, shorter stems, and delayed reproductive events than in wild-type (WT) plants. In transgenic switchgrass, expression of $P v G A 2$ ox 5 and $P v G A 20 x 9$ improves architecture and reduces biomass recalcitrance.

Submitted 8 August 2017, last revision 11 November 2017, accepted 16 November 2017.

Abbreviations: ABA - abscisic acid; FLC - FLOWERING LOCUS C; FT- FLOWERING LOCUS T; GA - gibberellin; GA2oxs GA2-oxidases; $\mathrm{GA}_{3}$ - gibberellic acid; GUS - $\beta$-glucuronidase; IAA - indole-3-acetic acid; MeJA - methyl jasmonate; MS - Murashige and Skoog; PAC - paclobutrazol; qPCR - quantitative polymerase chain reactions; SOC1 - SUPPRESSOR OF OVEREXPRESSION OF CO1; WT - wild type.

Acknowledgements: We are very grateful to Dr. Y.-W. Jiang from Department of Agronomy, Purdue University and Miss Z.-S. Sun from School of Foreign Languages, Beihang University for critically reviewing the manuscript. This research was supported by the China Postdoctoral Science Foundation (2017M620677), the Beijing Postdoctoral Research Foundation (No.2017ZZ-087), the Postdoctoral Fund of the Beijing Academy of Agriculture and Forestry Sciences, the National Natural Science Foundation of China (No. 31672477), and the Innovative Project of Beijing Academy of Agriculture and Forestry Sciences (KJCX20151202).

* Corresponding authors; e-mails: yinsx369@163.com; tengke.123@163.com 
Ectopic expression of ClGA2ox1 or ClGA2ox3 from camellia in Nicotiana tabacum induces dwarfism and smaller flowers. Accordingly, Arabidopsis overexpressing BnGA2ox6 from rapeseed shows dwarfism, late flowering, and enhanced chlorophyll accumulation. Previous studies demonstrated that GA2oxs could be valuable factors in plant architecture engineering breeding.

Poa pratensis (Kentucky bluegrass), a typical $\mathrm{C}_{3}$ cool season grass, is widely used in home lawns, landscaping, football pitches, and golf courses (Puyang et al. 2015).

\section{Materials and methods}

Plants and growth conditions: Poa pratensis L. cv. Baron seeds were purchased from the TopGreen seed company (Beijing, China). The seedlings were cultivated in a greenhouse at day/night temperatures of $20 / 18{ }^{\circ} \mathrm{C}$, a relative humidity of approximately $60 \%$, a 12-h photoperiod, and an irradiance of $400 \mu \mathrm{mol} \mathrm{m} \mathrm{m}^{-2} \mathrm{~s}^{-1}$. Nicotiana benthamiana Domin was cultivated in a growth chamber at temperatures of $22 / 20^{\circ} \mathrm{C}$, a relative humidity of $65 \%$, a $16-\mathrm{h}$ photoperiod, and an irradiance of $200 \mu \mathrm{mol} \mathrm{m} \mathrm{m}^{-2} \mathrm{~s}^{-1}$. Arabidopsis thaliana L. ecotype Columbia plants were kept at day/night temperatures of $24 / 22{ }^{\circ} \mathrm{C}$, a relative humidity of $65 \%$, a $16-\mathrm{h}$ photoperiod, and an irradiance of $200 \mu \mathrm{mol} \mathrm{m} \mathrm{m}^{-2} \mathrm{~s}^{-1}$ to generate transgenic lines. Seeds utilized for the phenotype observation were harvested from individual lines, which grew under the identical growth condition. The plants were fertilized weekly using a half-strength Hoagland's solution (Hoagland and Arnon 1950).

Isolation of $\boldsymbol{P p} \boldsymbol{G A 2 o x}$ : Total RNA was isolated from $P$. pratensis leaves using the plant RNA kit (OMEGA, Norcross, GA, USA). Full-length 3'-5' sequences of PpGA2ox were obtained using rapid amplification of cDNA end (RACE) with a SMARTer RACE kit (Clontech, Palo Alto, CA, USA). Primer sequences for 3'-5' for RACE amplification were designed using a known cDNA sequence fragment screened from $R N A$-seq database (Table 1 Suppl.). The PCR products were purified and then inserted into the pMD19-T cloning vector (TaKaRa, Dalian, China) before being sequenced at Tianyi Huiyuan Company (Beijing, China). The obtained sequences were used to design specific primers, PpGA2ox-F and PpGA2ox-R, for amplification of fulllength complementary DNA and genomic DNA sequences.

Isolation of the PpGA2ox promoter: Genomic DNA was extracted from leaves using a plant DNA kit $(O M E G A)$. Using the gDNA as a template, the genome walking was carried out with a genome walking kit (TaKaRa) according to the instructions. Three specific primers, GA2ox-R1, GA2ox-R2, and GA2ox-R3, were
Semi-dwarf or dwarf $P$. pratensis is becoming increasingly popular around the world to save the intensive labour and energy costs in turf maintenance. GA2oxs were reported to fine tune the endogens GA content resulting in dwarf phenotypes of plants. However, little has been known about the GA2oxs in $P$. pratensis. The objectives of this study were to isolate a gibberellin 2-oxidase gene from $P$. pratensis, to determine its promoter activity and its subcellular localization pattern, and finally, to uncover its function utilizing transgenic Arabidopsis.

used. The purified PCR products were sequenced using the GA2ox-R3 primer. Based on the sequencing data, the promoter-specific primers, Prom-F and Prom-R, were used to amplify the upstream sequence of PpGA2ox.

Bioinformatics: The PpGA2ox amino acid sequence was derived from the corresponding cDNA sequence using $D N A M A N$ software (v. 7.0). BLAST analysis of the NCBI database was used to identify homologs. A phylogenetic tree was built using $M E G A$ (v. 5.0) with the neighborjoining method (Tamura et al. 2011). A bootstrap analysis was performed with 1000 replicates excluding positions with gaps. The molecular masses $(\mathrm{Mr})$ and theoretical isoelectric points $(\mathrm{pI})$, potential signal peptide cleavage sites, subcellular localization pattern, and cis-regulatory elements in promoter were analyzed by Compute $\quad \mathrm{pI} / \mathrm{MW}$ tool (http://web.expasy.org/ compute_pi/), SignalP 4.1 server (http://www.cbs.dtu. $\mathrm{dk} /$ services/SignalP/) (Petersen et al. 2011), ProtComp 9.0 (http://www.softberry.com), and PlantCARE database (http://bioinformatics.psb.ugent.be/webtools/plantcare/ht $\mathrm{ml} /$ ), respectively.

Real-time quantitative PCR: The expression pattern of PpGA2ox in $P$. pratensis was analyzed in the leaves at different developmental stages: young (3-week-old), fastgrowing (6-week-old), and mature (3-month-old), as well as in roots and stems using real-time qPCR. PpGA2ox expression profiles were examined in 3-month-old $P$. pratensis after $24-\mathrm{h}$ induction with $10 \mu \mathrm{M}$ gibberellic acid $\left(\mathrm{GA}_{3}\right), 5 \mu \mathrm{M}$ methyl jasmonate (MeJA), or $10 \mu \mathrm{M}$ indole-3-acetic acid (IAA). GUS gene expression analysis was determined in 2-week-old transgenic A. thaliana under treatments with $10 \mu \mathrm{M} \mathrm{GA}$ or $10 \mu \mathrm{M}$ paclobutrazol (PAC). Arabidopsis FLOWERING LOCUS C (AtFLC), SUPPRESSOR OF OVER-EXPRESSION OF CO1 (AtSOC1), and FLOWERING LOCUS T (AtFT) were selected to monitor the flowering mechanisms. All the gene specific primers could be found in Table 1 Suppl. Reactions were performed in 96-well blocks with a CFX Connect PCR system (BIO-RAD, Hercules, CA, USA) using SYBR Premix (TaKaRa) in a total volume of 
$0.025 \mathrm{~cm}^{3}$. The two-step program was set: initial denaturation at $95{ }^{\circ} \mathrm{C}$ for $30 \mathrm{~s}$, followed by 40 cycles of $95^{\circ} \mathrm{C}$ for $5 \mathrm{~s}$, and $60{ }^{\circ} \mathrm{C}$ for $30 \mathrm{~s}$. The $P$. pratensis actin (GenBank accession No. KX342945) and $A$. thaliana UBQ10 (GenBank accession No. NM116771) were selected as the internal reference genes (Table 1 Suppl.). The relative expression was calculated using the comparative $\Delta \Delta \mathrm{Ct}$ method (Livak and Schmittgen 2001). All data were presented as the means of at least three independent biological replicates (each including three technical replicates) with corresponding standard deviations (SDs).

Binary vector construction and generation of transgenic plants: The $35 S:: P p G A 20 x: Y F P$ fusion construct was created by inserting the complete $P p G A 2 o x$ open reading frame (ORF) into plasmid 3302Y(Jia et al. 2016). Primers 3302Y-GA2ox-F and 3302Y-GA2ox-R were used to amplify the PpGA2ox coding sequence (Table 1 Suppl.), which was then purified and inserted into $3302 \mathrm{Y}$ vector digested with $B g l \mathrm{II}$ (TaKaRa) using an In-fusion HD cloning kit (TaKaRa).

The $P p G A 2 o x_{\text {pro }}: \because G U S$ fusion construct contained a 1109 bp PpGA2ox promoter region. The promoter region was amplified from the plasmid containing the target sequence using primers 1391-GA2ox-F and 1391GA2ox-R (Table 1 Suppl.). The pCAMBIA1391Z vector was digested with NcoI (TaKaRa). Then the purified $P p G A 2 o x$ promoter was inserted into the digested vector using an In-fusion HD Cloning kit (TaKaRa) to produce the PpGA2ox pro $_{\text {P }}: G U S$ fusion construct.

Using the floral dip method, Agrobacterium strain GV3101 transformed with the constructed plasmids was used to infect Arabidopsis plants to generate transgenic plants expressing $P p G A 2 o x$ or $P p G A 2 o x_{p r o}:: G U S$. Transformed Arabidopsis plants $\left(\mathrm{T}_{2}\right)$ were screened using $60 \mathrm{mg} \mathrm{dm}^{-3}$ glufosinate. Positive transgenic plants were verified by PCR. Representative $\mathrm{T}_{3}$ transgenic lines that exhibited $100 \%$ resistance to glufosinate were harvested for further phenotype observation or GUS staining assays.

\section{Results}

The PpGA2ox cDNA sequence was deposited in the NCBI database with the accession number of KX254272. The ORF of PpGA2ox was 1047 bp in length, corresponding to 348 amino acids (Fig. 1A Suppl.). PpGA2ox belonged to the GA2ox superfamily. The theoretical pI was 6.21, and the molecular mass was $37.89 \mathrm{kDa}$. No potential signal peptide was found in the PpGA2ox protein. Phylogenetic analysis showed that PpGA2ox was most closely related to the proteins from Hordeum vulgare or Triticum aestivum (Fig. 1B Suppl.).

A 1109 bp upstream fragment in front of the ATG start codon containing CAAT-box and TATA-box was
Subcellular localization of PpGA2ox and GUS assay: To examine the subcellular localization pattern of PpGA2ox, Agrobacterium strain EHA105 transformed with the 35S::PpGA2ox:YFP fusion construct was used to transform 3-week old $N$. benthamiana plants according to the method of Sparkes et al. (2006). After dark inducement for $48 \mathrm{~h}$, tobacco leaf cells were examined and photographed using a $S P-5$ laser confocal scanning microscope (Leica, Mannheim, Germany) according to the method of Teng et al. (2016).

The GUS staining was carried out using a GUS kit (O'BioLab, Beijing, China) according to the instruction provided by the manufacturer. After removing the chlorophyll with $70 \%(\mathrm{v} / \mathrm{v})$ ethanol, the tobacco seedlings were photographed using a M205FA stereomicroscope (Leica, Mannheim, Germany).

Phenotype observation: Arabidopsis line-35 and line-36 with the highest amount of transcripts among the $\mathrm{T}_{3}$ transgenic lines were selected as the representative lines for the phenotype observation. The seeds of the representative lines and wild type (WT) were sterilized with $70 \% \quad(\mathrm{v} / \mathrm{v})$ ethanol and $1 \%(\mathrm{~m} / \mathrm{v})$ sodium hypochlorite and then sowed on Murashige and Skoog (MS) medium. The phenotype was photographed using an EOS 60D digital camera (Canon, Tokyo, Japan) after growing for $14 \mathrm{~d}$. The chlorophyll content was measured using in $95 \%$ ethanol extract according to the protocol of Teng et al. (2016). To examine whether exogenous application of gibberellin could rescue the dwarfism phenotype of the PpGA2ox-overexpressing lines, sterilized seeds of both the transgenic lines and WT were sowed on the MS medium in culture flasks with or without $\mathrm{GA}_{3}$. After 4 weeks, the seedlings were photographed. For the determination of flowering time, the 12-d-old seedlings of line-35 and line-36 were transplanted from MS medium to nutrition medium containing peat, Vermiculite, and Perlite $(1: 1: 1$ in volume) and grown in growth chambers until the primary open flowers were observed.

examined for promoter elements identification (Fig. $1 C$ Suppl.). Two MeJA-responsive motifs were also identified, including CGTCA-motif and TGACG-motif. In addition, one cis-acting regulatory element related to meristem expression (CAT-box) and two involved in endosperm expression (GCN4-motif and Skn-1 motif) were found.

Real-time qPCR was carried out to investigate the expression characters of PpGA2ox. The transcripts were found in all investigated tissues including leaf, stem, and root, and the highest transcription was observed in the leaves (Fig. 1A). Moreover, the expression of PpGA2ox 
was higher in young leaves than in fast-growing and in mature leaves (Fig. $1 B$ ). The PpGA2ox expression was up-regulated significantly by $10 \mu \mathrm{M} \mathrm{GA}_{3}$ with the highest transcript abundance after $24 \mathrm{~h}$ (122-fold higher than before application; Fig. $1 C$ ). In addition, PpGA2ox was induced by $5 \mu \mathrm{M}$ MeJA or $10 \mu \mathrm{M}$ IAA, with the highest expression found after 6 and $1 \mathrm{~h}$, respectively (Fig. 1D,E).

Transient overexpression of $35 S:: P p G A 2 o x: Y F P$ in $N$. benthamiana leaf cells allowed us to study the subcellular localization of PpGA2ox. The strong yellow florescent protein (YFP) signal was detected in the whole cell of the control plants whereas the YFP signal was only found in nucleus and cytoplasm in 35S::PpGA2ox:YFP overexpressing cells (Fig. 2). It indicated that PpGA2ox was localized in nucleus and cytoplasm.

The native promoter of $P p G A 2 o x$ was infused with
GUS gene to generate the PpGA2oxpro::GUS construct. Then transgenic Arabidopsis lines were generated to explore the promoter activity. Histochemical analysis showed that strong GUS activity was in both the leaf apex and shoot apical region of the PpGA2oxpro::GUS transgenic seedlings (Fig. 3B-D). However, no GUS signal was found in the seedlings transformed with pCambia1391Z empty vector (the control) (Fig. 3A). It could be supposed that $P p G A 2 o x$ expressed most abundantly in leaf apex and shoot apical region.

Furthermore, real-time qPCR analysis was also employed to investigate the GUS gene expression under the treatments with $10 \mu \mathrm{M} \mathrm{GA}_{3}$ or $10 \mu \mathrm{M}$ PAC using the PpGA2oxpro::GUS transgenic seedlings. It showed that the GUS expression was 1.92-fold higher than control under $\mathrm{GA}_{3}$ treatment for $3 \mathrm{~d}$. However, it was lower than control after PAC inducement (Fig. 3 Suppl.).

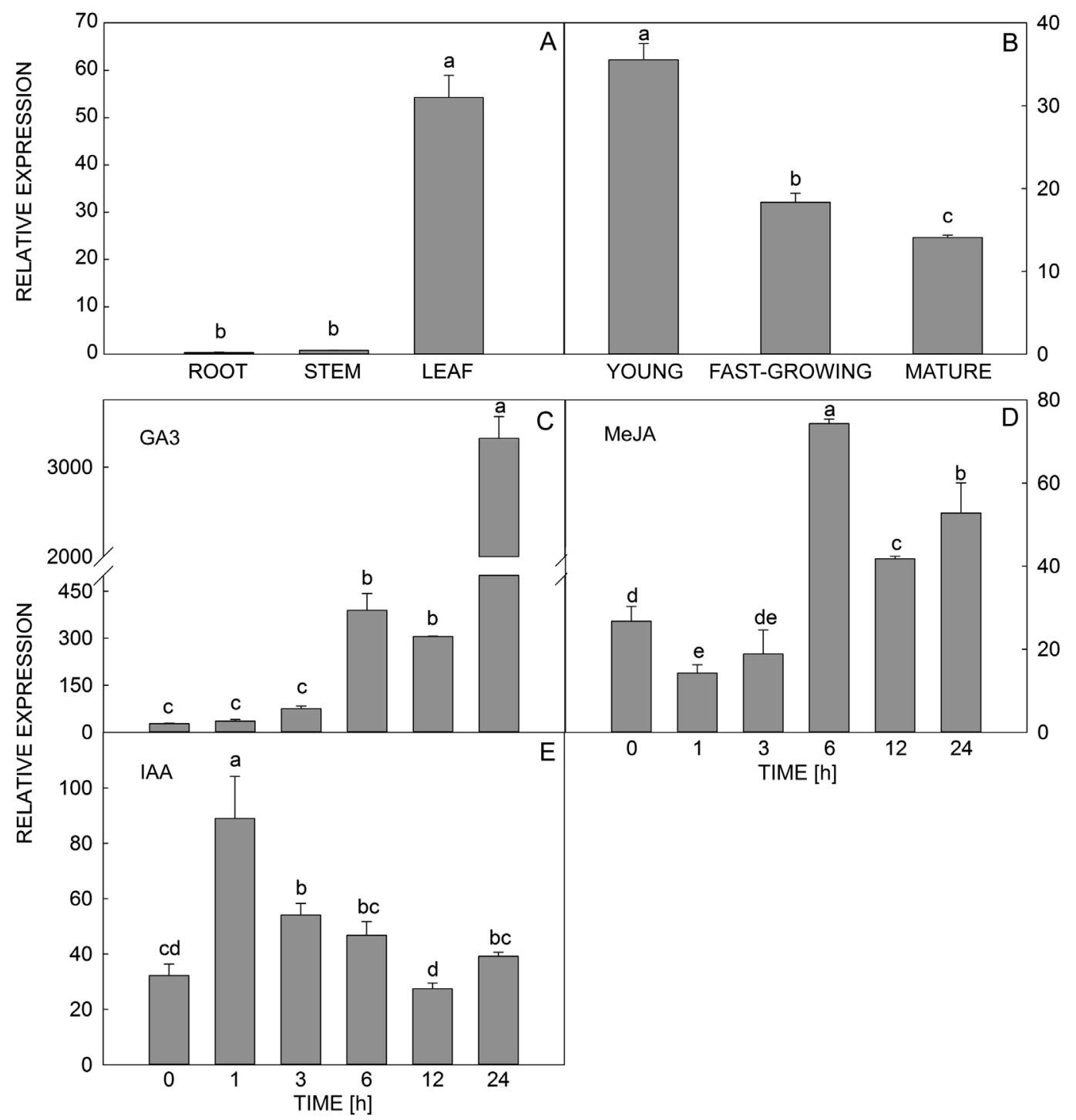

Fig. 1. Expression profiles of PpGA2ox. A - PpGA2ox expression in root, stem, and leaf of Poa pratensis. $B$ - Expression of $P p G A 2 o x$ in leaves at different developmental stages. $C, D, E-P p G A 2 o x$ expression in plants exposed to $10 \mu \mathrm{M} \mathrm{GA}_{3}(C), 5 \mu \mathrm{M}$ $\operatorname{MeJA}(D)$, and $10 \mu \mathrm{M}$ IAA $(E)$. Means \pm SDs $(n=3)$. Different letters indicate significant differences at $5 \%$ level of probability. 
Transgenic Arabidopsis plants overexpressing $P p G A 2 o x$ were generated to further explore the function of PpGA2ox. Two representative $\mathrm{T}_{3}$ homozygous lines, line-35 and line-36, were selected based on their higher expression of PpGA2ox than in other lines. Transgenic Arabidopsis seedlings exhibited gibberellin deficiency leading to dwarf phenotype with smaller leaves and shorter stems compared with WT after being grown on the MS medium for $14 \mathrm{~d}$ (Fig. 4A,B). The fresh mass of the transgenic lines was also lower than that of WT (data not shown).

To investigate the underlying genetic mechanism that caused the dwarfism of the transgenic lines, the relative expressions of AtGA2ox1, AtGA2ox2, and AtGA2ox3 were
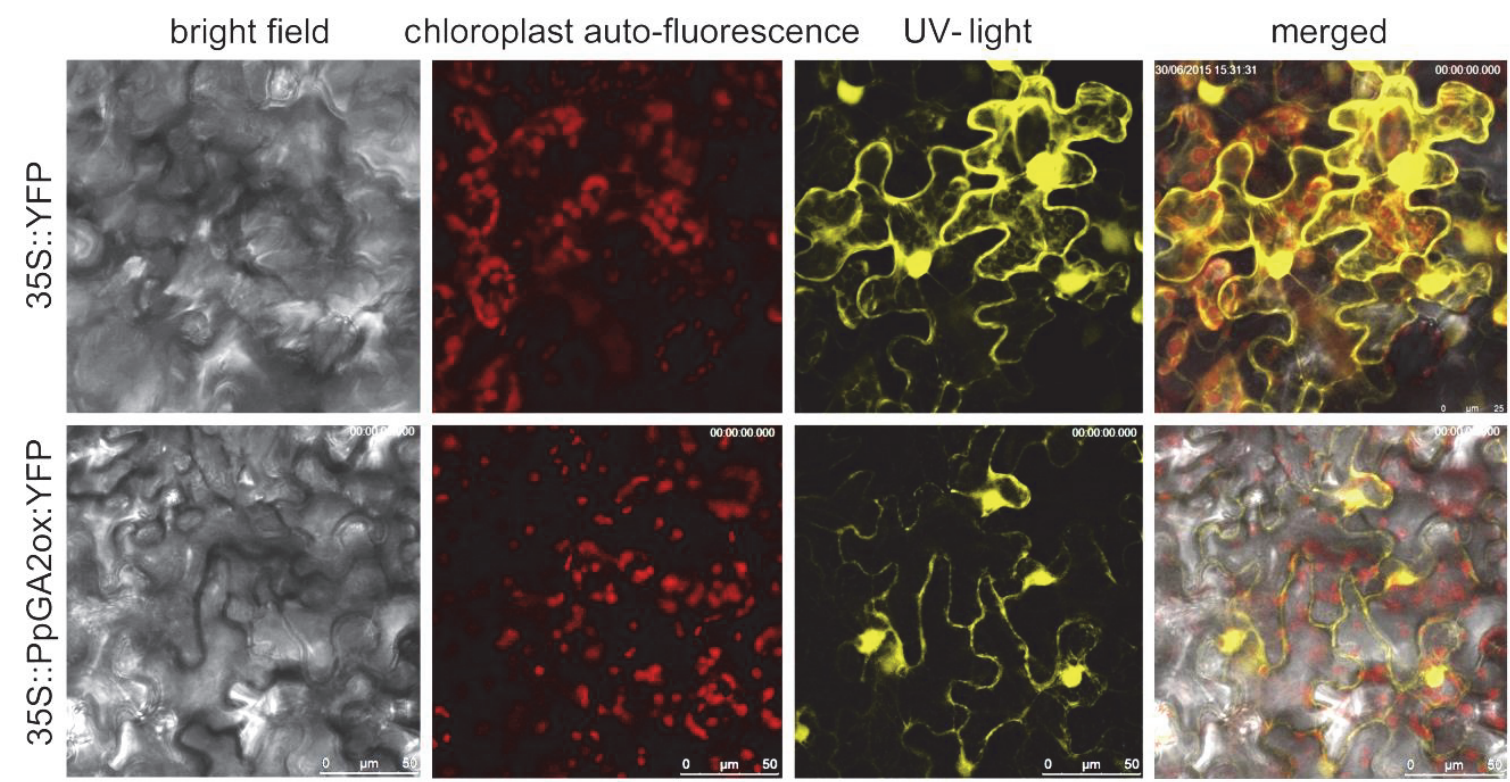

Fig. 2. Subcellular localization of PpGA2ox under UV radiation in Nicotiana benthamiana leaf epidermis. Fluorescence due to the 35S:: PpGA2ox:YFP was strongly distributed in both the nucleus and cytoplasm. The scale bar is $50 \mu \mathrm{m}$.
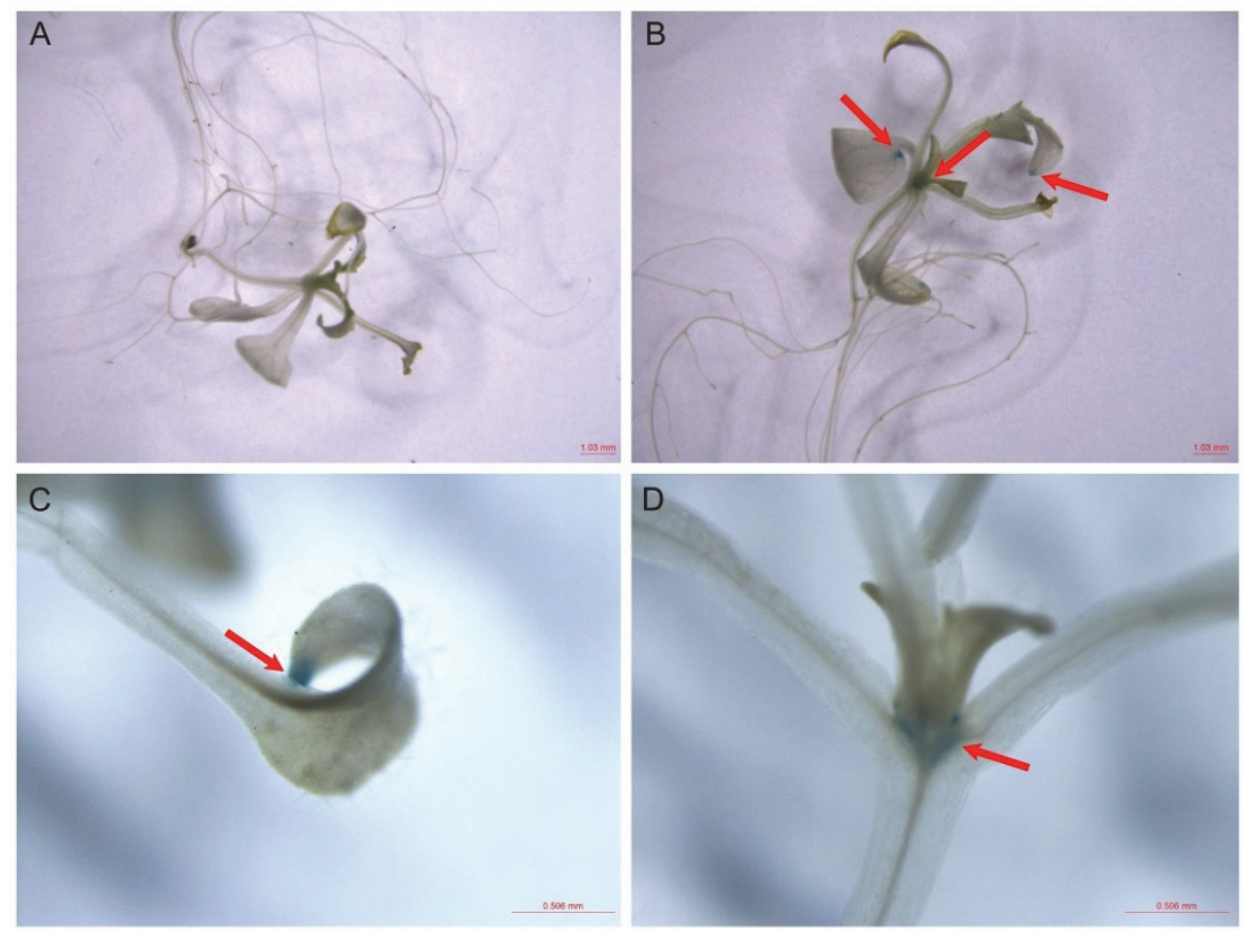

Fig. 3. GUS staining (blue colour) in 2-week-old Arabidopsis seedlings: control (A), PpGA2ox ${ }_{P r o}:$ GUS-overexpressing lines (B), leaf apex $(C)$, and shoot apical region $(D)$. The scale bar is $1 \mathrm{~mm}(A, B)$ or $0.5 \mathrm{~mm}(C, D)$. 
examined using real-time qPCR. The results showed that the expression of the selected marker genes related to gibberellin catabolism were higher in the transgenic lines than in the WT plants (Fig. 4C-F). In particular, the relative expressions of AtGA2ox1, AtGA2ox2, and AtGA2ox3 in line-35 increased by $117.8,68.3$, or $31.7 \%$ compared to WT, respectively.

After 4 weeks of cultivation on MS medium without $\mathrm{GA}_{3}$, line-35 and line-36 exhibited obvious dwarf phenotype compared with the WT grown in MS medium without $\mathrm{GA}_{3}$ (Fig. 2 Suppl.). Nevertheless, the plant height difference between the transgenic lines and WT disappeared when they were grown on MS medium containing $\mathrm{GA}_{3}$, indicating that application of $100 \mu \mathrm{M}$ $\mathrm{GA}_{3}$ rescued the dwarf phenotype in a period of 4 weeks.

A surprising phenotype which PpGA2ox-overexpressing lines exhibited later flowering than the control drew our attention (Fig. 5A). To quantify the flowering time, we recorded the time when the first open flowers were observed and we found that overexpression of PpGA2ox delayed flowering for 10 to $14 \mathrm{~d}$ in comparison with WT plants. Three marker genes related to plant flowering time including AtFLC, AtSOC1, and AtFT were selected to monitor the underlying mechanisms. The

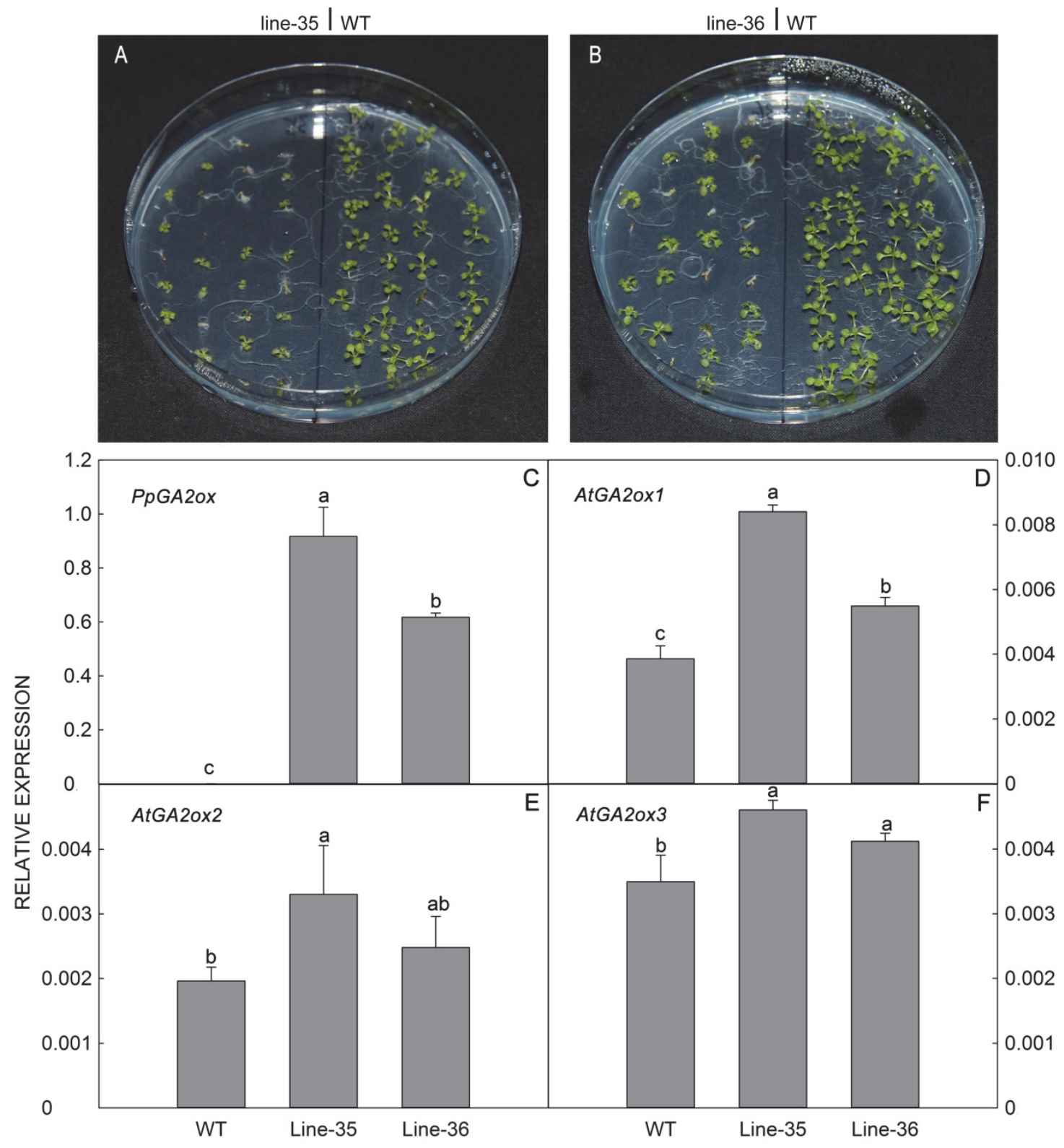

Fig. 4. Phenotype observation of 2-week-old $P p G A 2 o x$-overexpressing lines $35(A)$ and $36(B)$, Relative expressions of $P p G A 2 o x$ (C), AtGA2ox1 (D), AtGA2ox2 (E), and AtGA2ox3 (F). Means \pm SDs $(n=3)$. Different letters indicate significant differences at $5 \%$ level of probability. 
results showed that the relative transcription of $A t F L C$ in line-35 or line-36 was increased by 83.1 and $28.3 \%$ compared to that of WT, respectively (Fig. $5 D$ ). On the contrary, the expression of AtSOC1 in line-35 and line-36 was reduced by 41.6 or $31.0 \%$, respectively, and expression of AtFT was reduced by 77.6 or $63.6 \%$, respectively, relative to control (Fig. 5E,F). Moreover, the leaf colour of the transgenic lines was greener than that of WT but the leaf size was obviously smaller than in the WT (Fig. 5B). Chlorophyll content in line-35 and line-36 was 1.57- or 1.55-fold higher than in the WT (Fig. 5C).

\section{Discussion}

The GA2oxs, a kind of 2-oxoglutarate-dependent dioxygenases, catalyses the deactivation of bioactive GAs or their precursors. In this study, a novel gibberellin 2-oxidase gene, $P p G A 2 o x$, was isolated from $P$. pratensis with the aim of genetic engineering $P$. pratensis for improved architecture. PpGA2ox belonged to the GA2ox superfamily and contained three $\mathrm{Fe}^{2+}$ binding sites and one 2-oxoglutarate binding site. It was localized in both nucleus and cytoplasm which was consistent with the localization AtGA2ox4 in Arabidopsis (Lee et al. 2014), OsGA2ox6 in rice (Ueguchi-Tanaka et al. 2005), and
PslGA2ox in plum (El-Sharkawy et al. 2012). Previous studies proved that GA2oxs play diverse roles in regulating gibberellin metabolism compared with the cytosol localized GA20ox and GA3ox (Hedden and Phillips 2000, Olszewski et al. 2002, Wuddineh et al. 2015).

Examination of $P p G A 2 o x$ expression revealed that $P p G A 2 o x$ was widely distributed in different tissues of $P$. pratensis, but the highest expression was in leaves. In addition, $P p G A 2 o x$ was expressed most abundantly in young leaves, implying that $P p G A 2 o x$ actively
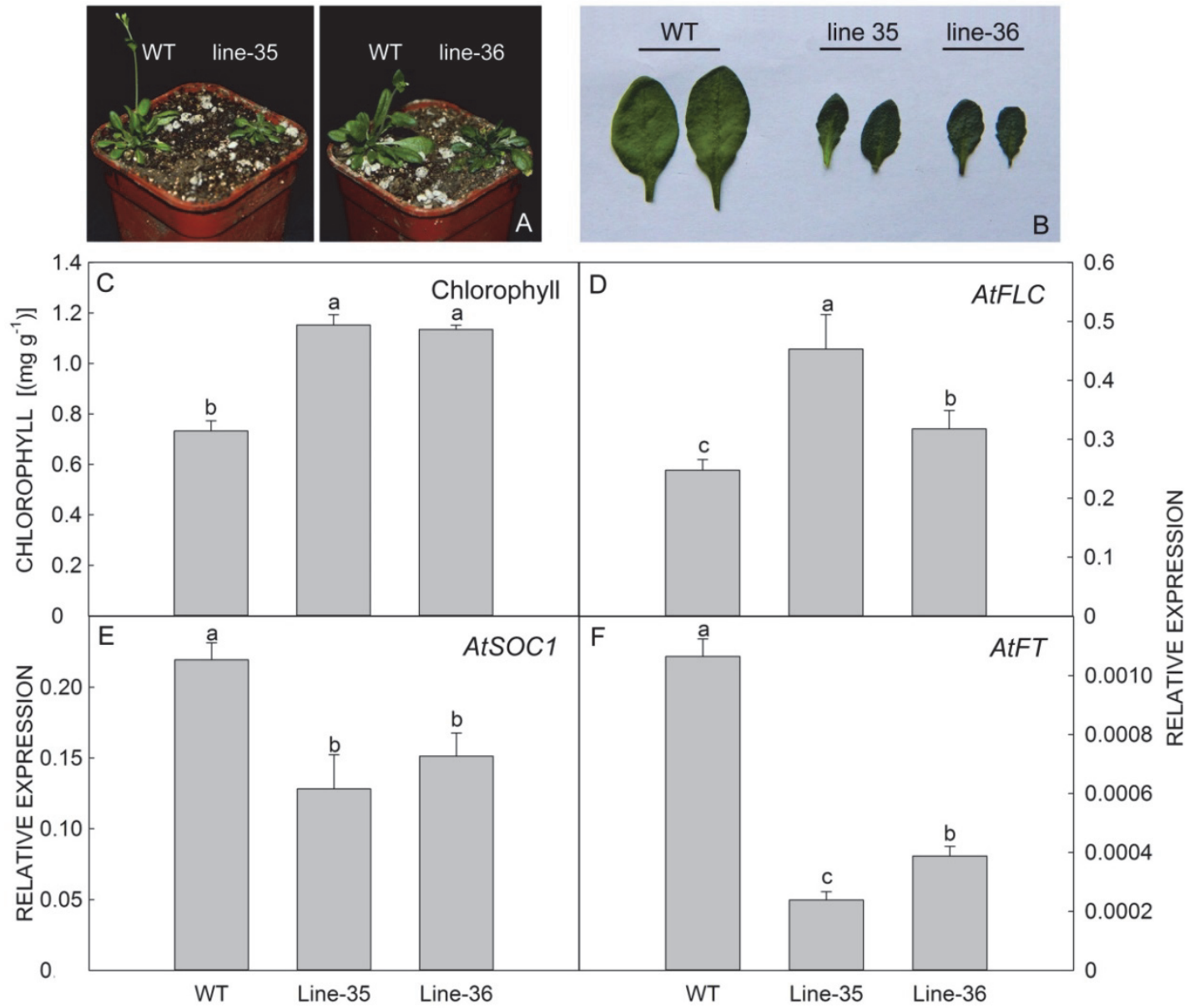

Fig. 5. $A$ - phenotype of WT and transgenic Arabidopsis plants line-35 and line-36. B, $C$ - Leaf colour $(B)$ and chlorophyll content per fresh mass basis $(C)$ in WT and transgenic plants. $D, E, F$ - Relative expressions of genes regulating flowering time: AtFLC (D), $\operatorname{AtSOCl}(E)$, and $\operatorname{AtFT}(F)$. Means \pm SDs $(n=3)$. Different letters indicate significant differences at a $5 \%$ level of probability. 
participated in early leaf development. The motifs identified in PpGA2ox promoter implied that its expression might be regulated by different hormones. Application of $10 \mu \mathrm{M} \mathrm{GA}_{3}$ induced the significant expression of $P p G A 2 o x$ in this study. It is consistent with the expression pattern of AaGA2ox1, AaGA2ox2 and AaGA2ox3 from Artocarpus altilis (Zhou and Underhill 2016). Exogenous $\mathrm{GA}_{3}$ might cause drastic fluctuation of endogenous GA due to activation of the GA biosynthetic and degradation mechanisms. Induction experiments proved that $P p G A 2 o x$ could be up-regulated by $5 \mu \mathrm{M}$ MeJA, which is supported by the MeJA-responsiveness cis-elements in the promoter sequence. The often reported regulation of GA by auxin led us to examine the expression of $P p G A 2 o x$ after IAA application. In this study, IAA application induced the $P p G A 2 o x$ expression during the first hour which is in accordance with the findings of Frigerio et al. (2006) that auxin up-regulates GA2ox expression very quickly after application.

To gain insight into the actual localization of $P p G A 2 o x$ in regulating GA metabolism in plant, we examined the spatial regulation of $P p G A 2 o x$ using GUS staining. Histochemical staining of GUS activity in transgenic Arabidopsis confirmed that PpGA2ox was expressed in both leaf apex and shoot apical region. This is in accordance with AtGA2ox2 (Frigerio et al. 2006). Further, real-time qPCR revealed that exogenous GA enhanced the expression of $G U S$ while PAC application caused a decline in the GUS expression. Together with the relative expression character of $P p G A 2 o x$ under GA treatment, it is rational to rule out a scenario that plants could activate the expression of $P p G A 2 o x$ to deal with the abrupt increase of GA, but suppress the expression of PpGA2ox to respond to the decrease of GA. This finding not only uncovered the actual functional localization of PpGA2ox in plants, but also interpreted the possible physiological roles of $P p G A 2 o x$ in regulating GA metabolism.

GA2oxs promote the inactivation of bioactive GAs or their precursors. Previous studies reported that GA2oxs overexpression resulted in typical GA-deficiency phenotype in plants (Wuddineh et al. 2015, Xiao et al. 2016, Yan et al. 2017). In this study, overexpression of PpGA2ox caused a dwarf phenotype in Arabidopsis.
AtGA2ox1, AtGA2ox2, and AtGA2ox3 were reported to generate inactive GA forms by catalyzing their $2 \beta$ hydroxylation (Olszewski et al. 2002, Gallego-Giraldo et al. 2008, Yamaguchi 2008, Hedden and Thomas 2012). The relatively high expressions of AtGA2ox1, AtGA2ox2, and $A t G A 20 x 3$ reflected a programed GA catabolism at least partially contributing to the dwarf phenotype of PpGA2ox-overexpressing plants. Furthermore, the dwarf phenotype of the transgenic lines was reversed by exogenous $\mathrm{GA}_{3}$. It supported the hypothesis that it was the overexpression of $P p G A 2 o x$ that inactivated the bioactive GAs in transgenic lines and resulted in the GA-deficiency leading to dwarfism.

Ectopic expressions of BnGA2ox6 (Yan et al. 2017), ClGA2oxl (Xiao et al. 2016), and NtGA2ox (Gargul et al. 2013) caused late flowering in A. thaliana, N. tabacum, and Petunia hybrid, respectively. Consistent with these results, the heterologous expression of $P p G A 2 o x$ caused delayed flowering time in transgenic Arabidopsis compared with that of the control. SOC1 (Hepworth et al. 2002) and FT (Hisamatsu and King 2008) were reported as key genes in accelerating flowering time, while FLC (Kim et al. 2007) was reported as a floral repressor. The relatively higher amount of $F L C$ transcripts together with the relatively lower amount of $S O C 1$ and $F T$ transcripts in $P p G A 20 x$-overexpressing lines interpreted the mechanism of the delayed flowering phenotype. In addition, the transgenic lines also showed dark green and small leaves, indicating a potential role of PpGA2ox in regulating chlorophyll biosynthesis and leaf development. The increased chlorophyll content in transgenic Arabidopsis lines was supported by the similar findings in plants overexpressing BnGA2ox6 (Yan et al. 2017), and ClGA2oxl and ClGA2ox3 (Xiao et al. 2016).

In conclusion, $P p G A 2 o x$ was successfully isolated from $P$. pratensis. Its expression was most abundant in young leaves. PpGA2ox was localized in both nucleus and cytoplasm and its native promoter could drive GUS expression in both the leaf apex and shoot apical region. Overexpression of PpGA2ox caused GA-deficient phenotype in Arabidopsis including dwarf plants, delayed flowering time, and increased chlorophyll content. Our study suggested that PpGA2ox could be a valuable gene for breeding new cultivars of $P$. pratensis.

\section{References}

El-Sharkawy I., El Kayal, W., Prasath, D., Fernandez, H., Bouzayen, M., Svircev, A.M., Jayasankar, S.: Identification and genetic characterization of a gibberellin 2-oxidase gene that controls tree stature and reproductive growth in plum. J. exp. Bot. 63: 1225-1239, 2012.

Frigerio, M., Alabadi, D., Perez-Gomez, J., Garcia-Carcel, L., Phillips, A.L., Hedden, P., Blazquez, M.A.: Transcriptional regulation of gibberellin metabolism genes by auxin signaling in Arabidopsis. - Plant Physiol. 142: 553-563,

2006.

Gallego-Giraldo, L., Ubeda-Tomas, S., Gisbert, C., GarciaMartinez, J.L., Moritz, T., Lopez-Diaz, I.: Gibberellin homeostasis in tobacco is regulated by gibberellin metabolism genes with different gibberellin sensitivity. Plant Cell Physiol. 49: 679-690, 2008.

Gargul, J.M., Mibus, H., Serek, M.: Constitutive overexpression of Nicotiana GA(2)ox leads to compact phenotypes and delayed flowering in Kalanchoe blossfeldiana and Petunia 
hybrida. - Plant Cell Tissue Organ Cult. 115: 407-418, 2013.

Hedden, P., Phillips, A.L.: Gibberellin metabolism: new insights revealed by the genes. - Trends Plant Sci. 5: 523-530, 2000.

Hedden, P., Thomas, S.G.: Gibberellin biosynthesis and its regulation. - Biochem. J. 444: 11-25, 2012.

Hepworth, S.R., Valverde, F., Ravenscroft, D., Mouradov, A., Coupland, G.: Antagonistic regulation of flowering-time gene SOC1 by CONSTANS and FLC via separate promoter motifs. - EMBO J. 21: 4327-4337, 2002.

Hisamatsu, T., King, R.W.: The nature of floral signals in Arabidopsis. II. Roles for FLOWERING LOCUS T $(F T)$ and gibberellin. - J. exp. Bot. 59:3821-3829, 2008.

Hoagland, D.R., Arnon, D.I.: The water-culture method for growing plants without soil. - Calif. Agr. Exp. Stn. Circ. 347: 357-359, 1950.

Jia, N., Liu, X.M., Gao, H.B.: A DNA2 homolog is required for DNA damage repair, cell cycle regulation, and meristem maintenance in plants. - Plant Physiol. 171: 318-333, 2016.

Kim, S.Y., Park, B.S., Kwon, S.J., Kim, J., Lim, M.H., Park, Y.D., Kim, D.Y., Suh, S.C., Jin, Y.M., Ahn, J.H., Lee, Y.H.: Delayed flowering time in Arabidopsis and Brassica rapa by the overexpression of FLOWERING LOCUS C (FLC) homologs isolated from Chinese cabbage (Brassica rapa $\mathrm{L}$.: ssp. pekinensis). - Plant Cell Rep. 26: 327-336, 2007.

Lee, D.H., Lee, I.C., Kim, K.J., Kim, D.S., Na, H.J., Lee, I.J., Kang, S.M., Jeon, H.W., Le, P.Y., Ko, J.H.: Expression of gibberellin 2-oxidase 4 from Arabidopsis under the control of a senescence-associated promoter results in a dominant semi-dwarf plant with normal flowering. - J. Plant Biol. 57: 106-116, 2014.

Livak, K.J., Schmittgen, T.D.: Analysis of relative gene expression data using real-time quantitative PCR and the $2^{-\Delta \Delta \mathrm{Ct}}$ method. - Methods 25: 402-408, 2001.

Olszewski, N., Sun, T.P., Gubler, F.: Gibberellin signaling: biosynthesis, catabolism, and response pathways. - Plant Cell 14 (Suppl): S61-S80, 2002.

Petersen, T.N., Brunak, S., Von Heijne, G., Nielsen, H.: Signal P 4.0: discriminating signal peptides from transmembrane regions. - Nat. Methods 8: 785-786, 2011.

Puyang, X.H., An, M.Y., Han, L.B., Zhang, X.Z.: Protective effect of spermidine on salt stress induced oxidative damage in two Kentucky bluegrass (Poa pratensis L.) cultivars. Ecotox. Environ. Safety 117: 96-106, 2015.

Sparkes, I.A., Runions, J., Kearns, A., Hawes, C.: Rapid, transient expression of fluorescent fusion proteins in tobacco plants and generation of stably transformed plants. Natur. Protocols 1: 2019-2025, 2006.

Tamura, K., Peterson, D., Peterson, N., Stecher, G., Nei, M., Kumar, S.: MEGA5: molecular evolutionary genetics analysis using maximum likelihood, evolutionary distance, and maximum parsimony methods. - Mol. Biol. Evol. 28: 2731-2739, 2011.

Teng, K., Chang, Z.H., Li, X., Sun., X.B., Liang, X.H., Xu, L.X., Chao, Y.H., Han, L.B.: Functional and RNAsequencing analysis revealed expression of a novel staygreen gene from Zoysia japonica (ZjSGR) caused chlorophyll degradation and accelerated senescence in Arabidopsis. - Front. Plant Sci. 7: 1894, 2016.

Ueguchi-Tanaka, M., Ashikari, M., Nakajima, M., Itoh, H., Katoh, E., Kobayashi, M., Chow, T.Y., Hsing, Y.I.C., Kitano, H., Yamaguchi, I., Matsuoka, M.: Gibberellin insensitive dwarf1 encodes a soluble receptor for gibberellin. - Nature 437: 693-698, 2005.

Wuddineh, W.A., Mazarei, M., Zhang, J.Y., Poovaiah, C.R., Mann, D.G.J., Ziebell, A., Sykes, R.W., Davis, M.F., Udvardi, M.K., Stewart, C.N.: Identification and overexpression of gibberellin 2-oxidase (GA2ox) in switchgrass (Panicum virgatum L.) for improved plant architecture and reduced biomass recalcitrance. - Plant Biotechnol. J. 13: 636-647, 2015.

Xiao, Z., Fu, R.P., Li, J.Y., Fan, Z.Q., Yin, H.F.: Overexpression of the gibberellin 2-oxidase gene from Camellia lipoensis induces dwarfism and smaller flowers in Nicotiana tabacum. - Plant mol. Biol. Rep. 34: 182-191, 2016.

Yamaguchi, S.: Gibberellin metabolism and its regulation. Annu. Rev. Plant Biol. 59: 225-251, 2008.

Yan, J.D., Liao, X.Y., He, R.Q., Zhong, M., Feng, P.P., Li, X.M., Tang, D.Y., Liu, X.M., Zhao, X.Y.: Ectopic expression of GA 2-oxidase 6 from rapeseed (Brassica napus L.) causes dwarfism, late flowering and enhanced chlorophyll accumulation in Arabidopsis thaliana. - Plant Physiol. Biochem. 111: 10-19, 2017.

Yin, S., Zhou, S., Kong, X., Han, Y., Wang, W.: Altered gibberellin content affects growth and development in trasgenic tobacco lines overexpressing a wheat gene encoding F-box protein. - Biol. Plant. 61: 349-358, 2017.

Zhou, Y.C., Underhill, S.J.R.: Breadfruit (Artocarpus altilis) gibberellin 2-oxidase genes in stem elongation and abiotic stress response. - Plant Physiol. Biochem. 98: 81-88, 2016. 